\title{
Estudo da composição e caracterização dos cristais de zircônias Y-TZP para Odontologia
}

\author{
Composition and characterization study of dental Y-TZP zirconia ceramics
}

\begin{abstract}
Beatriz Curvello de Mendonça, ${ }^{1}$ William Matthew Negreiros, ${ }^{1}$ Maicon Sebold, ${ }^{1}$ Adriana Oliveira Carvalho, ${ }^{1}$ Marina Di Francescantonio, ${ }^{1}$ Marcelo Giannini ${ }^{1}$
${ }^{1}$ Departamento de Odontologia Restauradora, Faculdade de Odontologia de Piracicaba, Universidade Estadual de Campinas, Piracicaba, SP, Brasil

- Os autores declaram que não há conflito de interesse.
\end{abstract}

\section{Resumo}

Objetivo: este trabalho teve como objetivo analisar a composição, a morfologia e o tamanho dos cristais de dióxido de zircônio de cinco marcas comerciais de zircônias Y-TZP odontológicas. Material e Métodos: os materiais avaliados, Cercon (Dentsply); Katana (Kuraray Noritake Dental Inc.), Lava (3M ESPE); Vita In-Ceram (Vita Zahnfabrik) e IPS e.max ZirCAD (Ivoclar Vivadent) foram investigados quanto à composição e morfologia/tamanho dos cristais utilizando as análises de energia dispersiva de raios-X e microscopia eletrônica de varredura, respectivamente $(n=5)$. Resultados: 0 elemento químico zircônio foi identificado em todos os materiais e os cristais mostraram diferentes modos de aglomeração e tamanhos que variaram de 0,1 a 0,5 $\mu$ m. Conclusão: as zircônias não apresentaram diferenças composicionais, mas a morfologia e tamanho dos cristais variaram dependendo do tipo de zircônia.

Palavras-chave: Zircônio; Raios-X; Microscopia eletrônica de varredura.

\section{ABSTRACT}

Objective: the objective of this study was to analyze the composition, morphology and size of dental zirconium dioxide crystals of five commercial brands of YTZ-P dental zirconias. Material and Methods: the materials evaluated, Cercon (Dentsply); Katana (Kuraray Noritake Dental Inc.), Lava (3M ESPE); Vita In-Ceram (Vita Zahnfabrik) and IPS e.max ZirCAD (Ivoclar Vivadent) were investigated according to their composition and crystal morphology/size, using energy dispersive X-rays and scanning electron microscope analysis, respectively $(n=5)$. Results: the zirconium chemical element was identified in all materials and the crystals showed different clustering modes and sizes that varied between 0.1 and $0.5 \mu \mathrm{m}$. Conclusion: the zirconia ceramics did not present compositional differences, but the morphology and size of crystals varied depending on the type of zirconia.

Keywords: Zirconium: X-Rays; Scanning electron microscopy.

\section{Introdução}

A introdução do mecanismo de tenacificação por transformação de fase da zircônia parcialmente estabilizada em meados de 1970 motivou a comunidade científica a analisar as possíveis aplicabilidades biomédicas do material. ${ }^{1}$ A zircônia possui três fases cristalográficas: monoclínica, tetragonal e cúbica. Com adição de óxidos metálicos como o $\mathrm{MgO}, \mathrm{CaO}, \mathrm{CeO}_{2}$ e $\mathrm{Y}_{3} \mathrm{O}_{2}$, a zircônia possui "metaestabilidade" em temperatura ambiente, tornando-a extremamente resistente à fratura. ${ }^{2}$

A primeira proposta da utilização do óxido de zircônio para aplicações biomédicas foi em ortopedia para reposição de cabeças de fêmur. Entretanto, nos anos 2000, seu uso foi descontinuado devido à alta taxa de fraturas espontâneas in vivo. ${ }^{3}$ Paralelamente, enquanto os médicos ortopedistas descontinuavam o uso da zircônia, os cirurgiões-dentistas estavam descobrindo as vantagens do material protético em Odontologia, sendo a principal delas substituir a maioria das restaurações metalocerâmicas. ${ }^{3}$

Devido à biocompatibilidade, qualidades estéticas e propriedades mecânicas favoráveis, o material possui ampla aplicação como copings, pilares de implante, coroas monolíticas, infraestruturas para próteses parciais fixas e até mesmo implantes dentários. ${ }^{3-13}$ Além disso, a produção de tais restaurações tornou-se facilitada com a introdução de sistemas CAD/CAM, que permitiram aos técnicos e cirurgiões-dentistas entregarem os trabalhos protéticos mais rápidos e com qualidade. ${ }^{14}$

As zircônias Y-TZP (zircônia tetragonal policristalina estabilizada por ítrio) para Odontologia são consideradas materiais cerâmicos, embora não apresentam fase vítrea como as cerâmicas feldspáticas, à base de dissilicato de lítio ou leucita. A principal característica dos materiais à base de dióxido de zircônio $\left(\mathrm{ZrO}_{2}\right)$ é a presença apenas da fase cristalina, que determina sua adesividade aos cimentos resinosos, características ópticas e propriedades físicas. ${ }^{15}$ Várias empresas de produtos odontológicos produzem zircônias para restaurações protéticas, entretanto é importante saber se elas apresentam similares composições baseadas nas morfologias dos cristais de dióxido de zircônio. ${ }^{16} \mathrm{O}$ objetivo deste estudo foi avaliar a composição química de cinco marcas comerciais de zircônia YTZ-P, a morfologia e o tamanho dos cristais que compõem cada material.

\section{Material e Métodos}

Cinco marcas comerciais de zircônia Y-TZP foram estudadas: Cercon (Dentsply/Degudent, Hanau, Alemanha); Katana (Kuraray Noritake Dental Inc., Aichi, Japão), Lava (3M ESPE AG, Seefeld, Alemanha); Vita In-Ceram Zirconia (Vita Zahnfabrik, Bad Sackingen, Alemanha) e IPS e.max ZirCAD (Ivoclar Vivadent AG, Schaan, Leichtensten). Dez placas (1,5 mm de espessura, 5 $\mathrm{mm}$ de comprimento e $5 \mathrm{~mm}$ de largura) de cada um desses materiais foram preparadas e avaliadas quanto à composição e formato dos cristais de dióxido de zircônio.

A composição de cada zircônia foi analisada por energia dispersiva de raios-X (Noran Instruments, Middleton, WI, EUA). As placas foram montadas em stubs de material polimérico $(n=5)$ e cobertas com carbono (Desk II, Denton Vacuum, Moorestwon, NJ, EUA). O espectro de cada amostra foi obtido com tempo de leitura de 100 segundos (voltagem: $15 \mathrm{kV}$; dead time 20-25\%; dis- 
tância de trabalho: $20 \mathrm{~mm})$.

Para caracterização da morfologia e tamanho dos cristais das zircônias, placas dos materiais $(\mathrm{n}=5)$ foram fixadas em stubs metálicos e cobertos com ouro em aparelho metalizador (MED 010, Balzers AG, Balzers, Liechtenstein). As zircônias foram examinadas em microscópio eletrônico de varredura (Leo 435 VP, Leo Electron Microscopy Ltda., Cambridge, Reino Unido) e áreas representativas nas amostras foram fotografadas em magnificações de 5.000X e 20.000X.

\section{Resultados}

As composições das zircônias estão presentes na figura 1. Para todos os materiais foram detectados a presença do elemento químico zircônio e carbono. O zircônio na tabela periódica é um metal, enquanto o carbono é relacionado ao tratamento das amostras para a análise em energia dispersiva de raios-X (coberturas das amostras com carbono). Nenhum outro elemento químico foi detectado, devido à baixa concentração nestes materiais.
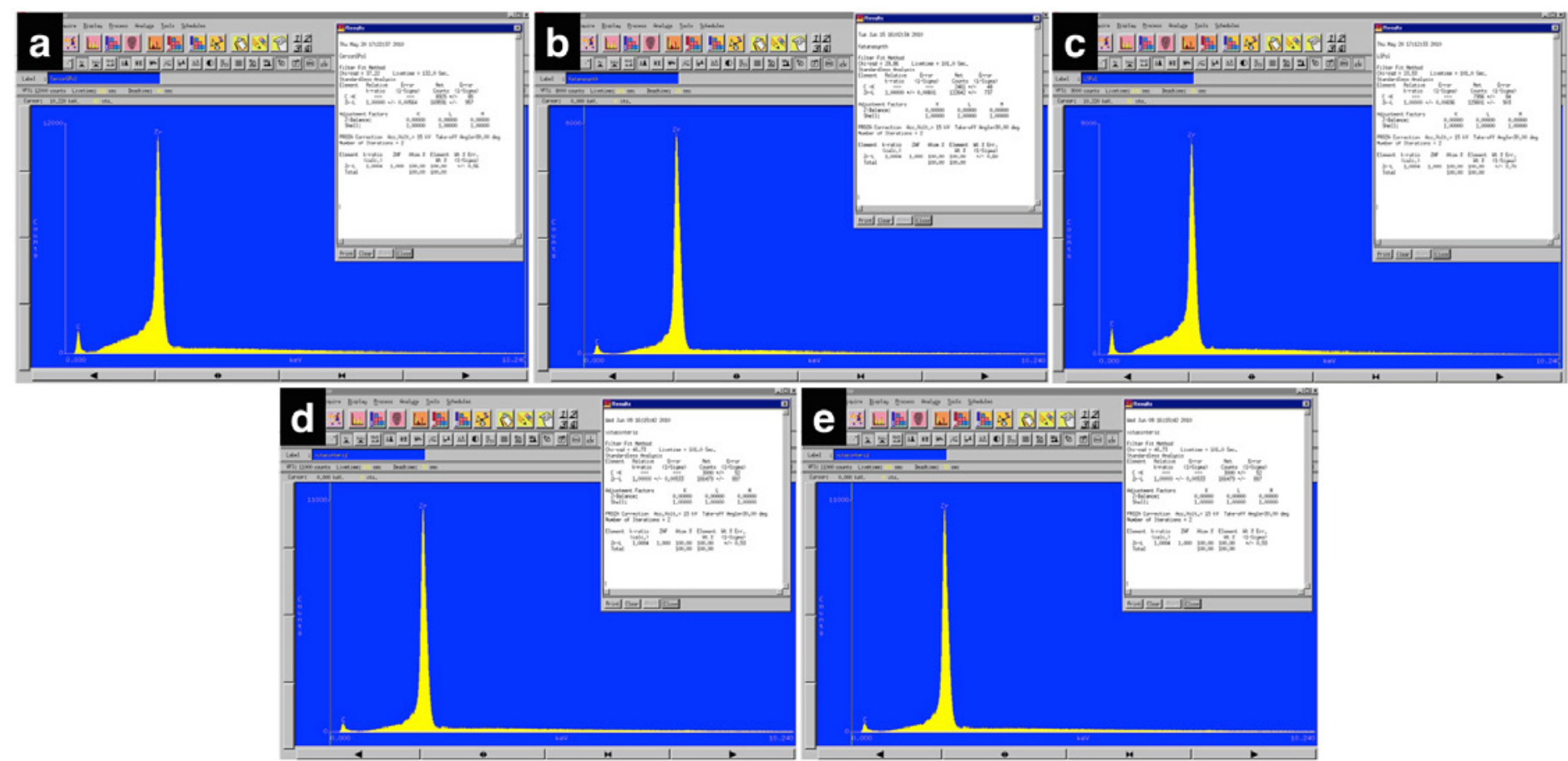

Figura 1. Elementos identificados pela análise de energia dispersiva de raios-X para as zircônias Cercon (1a), Katana (1b), Lava (1c); Vita In-Ceram Zirconia (1d) e IPS e.max ZirCAD (1e)

Com relação ao tamanho dos cristais de dióxido de zircônio (figuras 2 e 3), as zircônias Cercon e Lava mostraram partículas de 0,1 a 0,2 $\mu \mathrm{m}$, respectivamente, aglomeradas em "clusters" de 0,5 a $1 \mu \mathrm{m}$ (Figuras 2a, 2c, 3a e 3c). A zircônia Katana não apresentou aglomerados de partículas, sendo essas de tamanho aproximado de $0,2 \mu \mathrm{m}$ (figuras $2 \mathrm{~b}$ e $3 \mathrm{~b}$ ). As estruturas das zircônias Vita In-Ceram e IPS e.max ZirCAD parecem bem compactas com cristais de formatos definidos de tamanhos de 0,3 a $0,5 \mu \mathrm{m}$ e sem formação de aglomerados aparentes (figuras $2 \mathrm{~d}, 2 \mathrm{e}, 2 \mathrm{~d}$ e $3 \mathrm{e}$ ). 


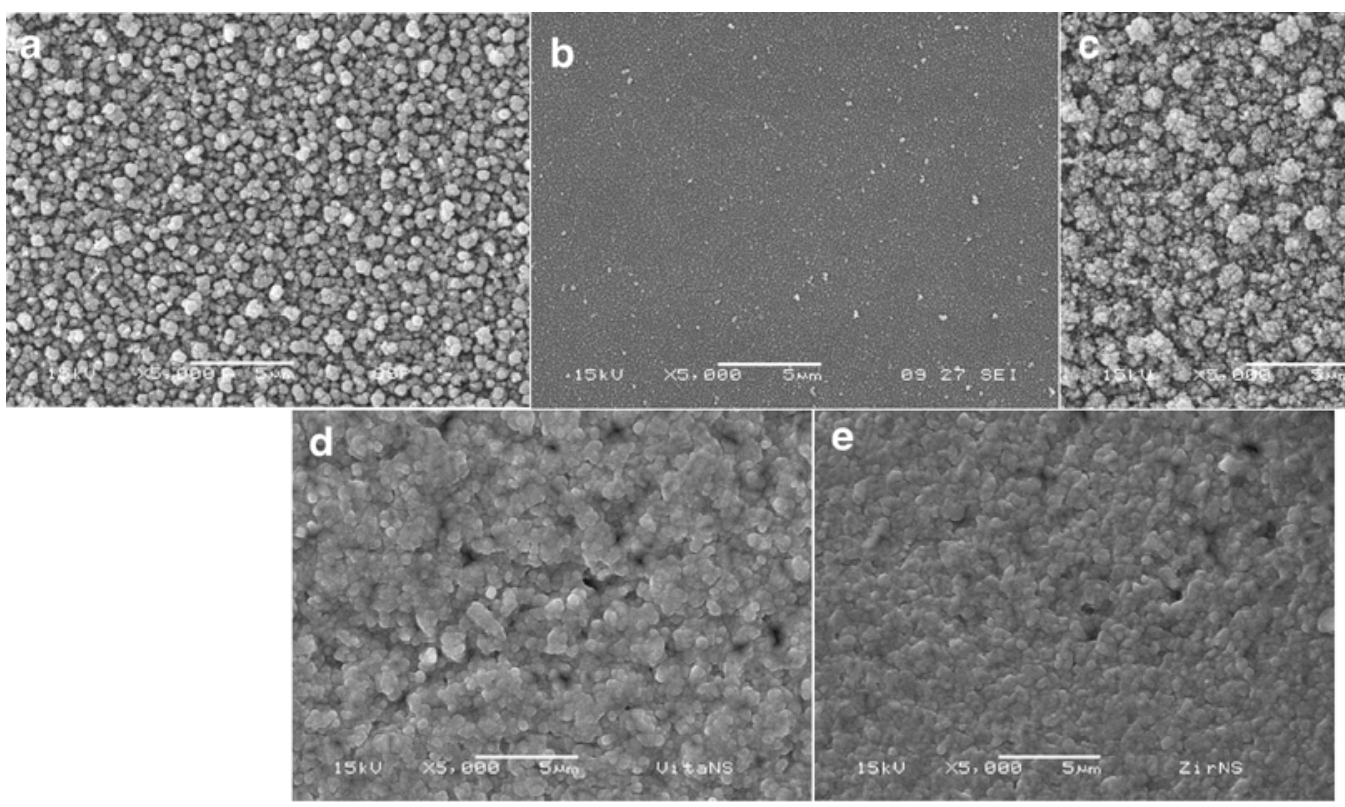

Figura 2. Microscopia eletrônica de varredura em 5.000x mostrando os cristais de dióxido de zircônio para as zircônias Cercon (2a), Katana (2b), Lava (2c); Vita In-Ceram Zirconia (2d) e IPS e.max ZirCAD (2e)

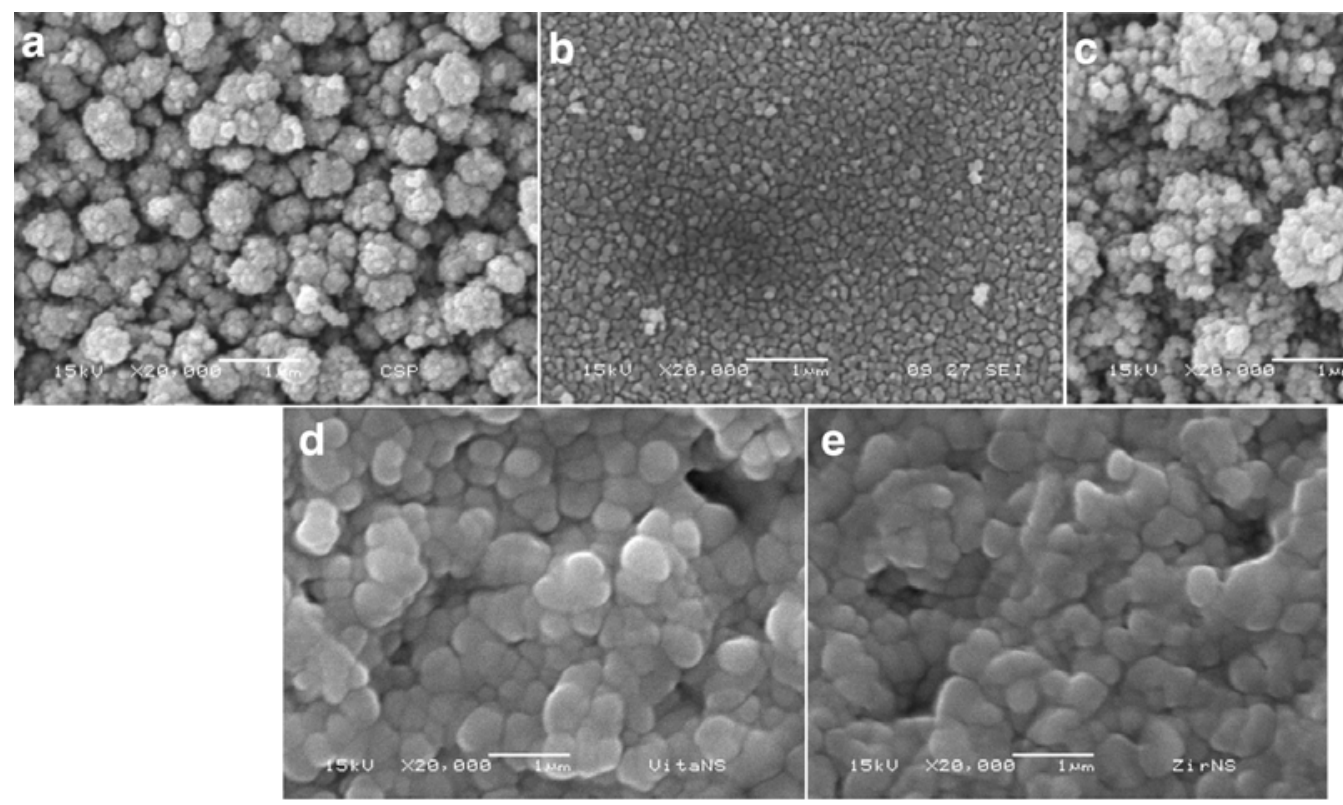

Figura 3. Microscopia eletrônica de varredura em 20.000x mostrando os cristais de dióxido de zircônio para as zircônias Cercon (3a), Katana (3b), Lava (3c); Vita In-Ceram Zirconia (3d) e IPS e.max ZirCAD (3e)

\section{Discussão}

A indicação da zircônia em trabalhos protéticos tem aumentado nos últimos e por isso é importante que os dentistas saibam as características e propriedades desses materiais, os quais empregam nas reabilitações dentais unitárias, parciais ou totais de seus pacientes. ${ }^{8-13}$ Muitas empresas têm produzido materiais à base de dióxido de zircônio, que se apresentam em blocos para CAD/CAM com diferentes tamanhos, cores e opacidades, sendo a maioria deles pré-sinterizados. ${ }^{16}$

Este estudo não identificou óxidos metálicos $\left(\mathrm{CaO}, \mathrm{MgO}, \mathrm{CeO}_{2}\right.$ e $\mathrm{Y}_{2} \mathrm{O}_{3}$ ), que são empregados para estabilizar o formato dos cristais, os quais ocorrem durante fenômenos de aquecimento e resfriamento. O principal óxido metálico adicionado à zircônia pura é o trióxido de ítrio, geralmente numa concentração de 3 a $6 \%$ (em peso $)^{2,15}$ e devido a essa baixa concentração ele não foi identificado na análise de energia dispersiva de raios-X.

O elemento químico zircônio foi encontrado em alta concentração para todos os materiais testados (figuras $1 \mathrm{~A}$ a $1 \mathrm{E}$ ), sendo $\mathrm{o}$ dióxido de zircônio o principal material presente em todos materiais testados. Segundo o fabricante do material Vita In-Ceram 
Zirconia, o óxido de alumínio $\left(\mathrm{Al}_{2} \mathrm{O}_{3}\right)$ é adicionado para proporcionar maior resistência,${ }^{15}$ mas também não foi identificado pela análise de energia dispersiva de raios-X.

A zircônia Cercon possui, segundo seu fabricante, óxido de ítrio (5\%), óxido de háfnio $(<3 \%)$, óxido de alumínio e de silício $(<1 \%)$, além do óxido de zircônio como principal componente. Para a zircônia Katana, o dióxido de zircônio está presente em $94,4 \%$, com $5,4 \%$ de óxido de ítrio e $0,2 \%$ de outros componentes em menores concentrações. A zircônia Lava apresenta 99\% de dióxido de zircônio, enquanto o IPS e.max ZirCAD também contém óxido de háfnio, óxido de alumínio e óxido de ítrio, além do dióxido de zircônio que é o componente em maior concentração. Todas essas informações sobre a composição das zircônias descritas acimas foram baseadas em informações fornecidas pelos fabricantes.

A zircônia possui três fases cristalinas muito distintas. A primeira delas é a monoclínica, que está presente em temperatura ambiente. Nessa fase cristalina, ela tem baixa resistência à flexão e devido à grande distância dos cristais, a zircônia mostra baixa dureza e pode ser facilmente usinada em unidades CAD/CAM. A partir de $1.170^{\circ} \mathrm{C}$ (temperatura inferior à maioria dos fornos de sinterização), os cristais têm formato tetragonal. Essa mudança de estrutura causa contração volumétrica que pode variar de $19 \%$ a 30\%, dependendo do estado inicial do bloco da zircônia, sendo ele pré-sinterizado ou não. Se o material for submetido a períodos mais prolongados de sinterização e/ou a temperatura de $2.370^{\circ} \mathrm{C}$, a estrutura cristalina do dióxido de zircônio se torna cúbica. Grãos cúbicos são muito maiores que os grãos monoclínicos e tetragonais, possuem elevada resistência flexural e dureza, porém são indesejados, pois desestabilizam os cristais adjacentes a ele..$^{2,15}$

Neste estudo não foi possível diferenciar o formato específico dos cristais destas fases cristalinas, mas foi possível determinar o tamanho deles, que variaram de 0,1 a $0,5 \mu \mathrm{m}$. Foram observados formações de aglomerados ou estruturas compactas, dependendo do material. Essas diferenças podem resultar em diferentes pro- priedades mecânicas, como resistência à flexão, módulo e dureza. ${ }^{5,15,16}$ Os aglomerados foram observados para as zircônias Cercon e Lava, enquanto estruturas compactas foram notadas para as zircônias Katana, Vita In-Ceram e IPS e.max ZirCAD.

As zircônias Cercon e Lava mostraram partículas aglomeradas em "clusters", sendo o tamanho dos cristais entre 0,1 a 0,2 $\mu \mathrm{m}$, respectivamente. Esses aglomerados de até $1 \mu \mathrm{m}$ de tamanho podem ter sido produzidos pela preparação das amostras para observação em microscopia. Os blocos de zircônia formam cortados para atingir o formato descrito anteriormente e tiveram um polimento inicial para planificação antes da sinterização e por causa desse processamento, talvez partículas se desprenderam e aglomeraram fora da massa compacta dessas zircônias. A zircônia Katana é o único material que se apresenta inicialmente como um bloco não pré-sinterizado. Esse material também teve partículas pequenas com tamanho aproximado de $0,2 \mu \mathrm{m}$. As maiores partículas foram observadas para as zircônias Vita In-Ceram e IPS e.max ZirCAD, com tamanho variável de 0,3 a 0,5 $\mu \mathrm{m}$. Devido ao tamanho dessas partículas foi possível observar melhor o formato dos grãos/cristais, que aparentam ter um formato mais definido e próximo do tetragonal.

\section{Conclusão}

A análise composicional não mostrou diferença entre as marcas comerciais de zircônia, sendo o elemento químico zircônio identificado em todos os materiais. Entretanto, a análise da morfologia e do tamanho dos cristais mostrou diferenças entre os materiais avaliados, com a presença de aglomerados e cristais compactados com tamanhos variados de 0,1 a $0,5 \mu \mathrm{m}$.

\section{Agradecimentos}

Este estudo teve suporte financeiro do SAE-PRP-UNICAMP (Bolsa de Iniciação Científica), do CNPq (307217-2014-0) e Capes (1777-2014).

\section{Referências}

1. Garvie RC, Hannink RH, Pascoe RT. Ceramic Steel. Nature. 1975;258(5537):703-4. 2. Piconi C, Maccauro G. Zirconia as a ceramic biomaterial. Biomaterials. 1999;20(1):1-25.

3. Chevalier J. What future for zirconia as a biomaterial? Biomaterials. 2006;27(4):535-43.

4. Manicone PF, Rossi Iommetti P, Raffaelli L, Paolantonio M, Rossi G, Berardi $\mathrm{D}$, et al. Biological considerations on the use of zirconia for dental devices. Int J Immunopathol Pharmacol. 2007;20(1 Suppl 1):9-12.

5. Manicone PF, Rossi Iommetti P, Raffaelli L. An overview of zirconia ceramics: basic properties and clinical applications. J Dent. 2007;35(11):819-26.

6. Oliva J, Oliva X, Oliva JD. Five-year Success Rate of 831 Consecutively Placed Zirconia Dental Implants in Humans: A Comparison of Three Different Rough Surfaces. Int J Oral Max Impl. 2010;25(2):336-44.

7. Oliva J, Oliva XV, Oliva JD. One-year follow-up of first consecutive 100 zirconia dental implants in humans: A comparison of 2 different rough surfaces. Int J Oral Max Impl. 2007;22(3):430-5.

8. Edelhoff D, Sorensen JA. Tooth structure removal associated with various preparation designs for posterior teeth. Int J Periodontics Restorative Dent. 2002;22(3):241-9.

9. Edelhoff D, Sorensen JA. Tooth structure removal associated with various preparation designs for anterior teeth. J Prosthet Dent. 2002;87(5):503-9.
10. Sailer I, Makarov NA, Thoma DS, Zwahlen M, Pjetursson BE. All-ceramic or metal-ceramic tooth-supported fixed dental prostheses (FDPs)? A systematic review of the survival and complication rates. Part I: Single crowns (SCs). Dent Mater. 2015;31(6):603-23.

11. Guncu MB, Cakan U, Muhtarogullari M, Canay S. Zirconia-based crowns up to 5 years in function: a retrospective clinical study and evaluation of prosthetic restorations and failures. Int J Prosthodont. 2015;28(2):152-7.

12. Tartaglia GM, Sidoti E, Sforza C. Seven-year prospective clinical study on zirconia-based single crowns and fixed dental prostheses. Clin Oral Investig. 2015;19(5):1137-45.

13. Abdulmajeed AA, Lim KG, Narhi TO, Cooper LF. Complete-arch implant-supported monolithic zirconia fixed dental prostheses: A systematic review. J Prosthet Dent. 2016;115(6):672-7.

14. Christensen JJ. Achieving optimal outcomes with all-zirconia crowns. Gen Dent. 2014 Jan-Feb;62(1):e6-9.

15. Cavalcanti AN, Foxton RM, Watson TF, Oliveira MT, Giannini M, Marchi GM. Y-TZP ceramics: key concepts for clinical application. Oper Dent. 2009;34(3):344-51.

16. Giordano R, McLaren EA. Ceramics overview: classification by microstructure and processing methods. Compend Contin Educ Dent. 2010;31(9):682-4. 


\section{Mini Currículo e Contribuição dos Autores}

1. Beatriz Curvello de Mendonça - cirurgiã-dentista e mestranda em Clínica Odontológica. Contribuição: análise microscópica, levantamento bibliográfico e preparação das figuras.

2. William Matthew Negreiros - cirurgião-dentista e mestre em Materiais Dentários. Contribuição: procedimento técnico, aquisição e análise dos dados e redação do manuscrito.

3. Maicon Sebold - cirurgião-dentista e mestrando em Clínica Odontológica. Contribuição: procedimento técnico, aquisição e análise dos dados.

4. Adriana Oliveira Carvalho - cirurgiã-dentista e doutora em Materiais Dentários. Contribuição: procedimento técnico, aquisição e análise dos dados.

5. Marina Di Francescantonio - cirurgiã-dentista e doutora em Clínica Odontológica. Contribuição: procedimento técnico, aquisição e análise dos dados.

6. Marcelo Giannini - cirurgião-dentista e doutor em Clínica Odontológica. Contribuição: Concepção e delineamento, análise dos dados e redação do artigo.

Recebido em: 08/11/2016 / Aprovado em: 03/12/2016

Autor Correspondente

Marcelo Giannini

E-mail: gianinni@unicamp.br 